\title{
Revista Biblioteca Universitaria: una década de evolución*
}

\author{
Felipe Rafael Reyna Espinosa**
}

Resumen: Al cumplir diez años la Nueva Época de la revista Biblioteca Universitaria se hace un recuento de su trayectoria, contenidos y temas tratados, así como del trabajo editorial que permitió elevar la difusión y la calidad de la revista, tanto en términos de criterios de calidad editorial, como en lo relacionado con el diseño gráfico y editorial. Biblioteca Universitaria publica principalmente artículos con contenido técnico profesional, especializada en bibliotecología y ciencia de la información, editada por la Dirección General de Bibliotecas (DGB) de la Universidad Nacional Autónoma de México (UNAM),

Palabras clave: revistas académicas, bibliotecología y ciencia de la información, universidades, criterios editoriales, trabajo editorial, revista Biblioteca Universitaria, publicación electrónica, Dirección General de Bibliotecas (DGB), UNAM, México.

\section{Biblioteca Universitaria Journal: a decade of editorial evolution}

Abstract: The New Series of the journal "Biblioteca Universitaria" is now 10 years old. This work provides a review of its trajectory, its contents and the topics it has covered, and discusses the editorial work that increased its circulation and quality in terms of editorial criteria and graphic and editorial design. "Biblioteca Universitaria" mainly publishes technical and professional articles related to Library and Information Science. The journal is published by the General Directorate for Libraries (DGB) of the National Autonomous University of Mexico (UNAM).

Keywords: scholarly journals, Library and Information Science, universities, editorial standards, editorial work, "Biblioteca Universitaria" journal, electronic publishing, General Directorate for Libraries (DGB), UNAM, Mexico.

* Una versión preliminar de este estudio fue presentada en el VI Encuentro de Editores de Revistas Científicas, La Habana, Cuba, 13-15 de noviembre de 2007.

*** Dirección General de Bibliotecas (DGB) de la Universidad Nacional Autónoma de México (UNAM), Circuito de la Investigación Científica, D.F. México.

Correo-e: frre@unam.mx.

Recibido: 5-5-08; 2. ${ }^{a}$ versión: 1-7-08. 


\section{Antecedentes}

Tradicionalmente la Universidad Nacional Autónoma de México (UNAM) ha estado a la vanguardia de la provisión de servicios bibliotecarios y de información en México. Actualmente (2008) la UNAM cuenta con el sistema bibliotecario universitario más grande de México y América Latina, compuesto por 142 bibliotecas, y 57 colecciones. Dentro de la estructura universitaria corresponde a la Dirección General de Bibliotecas (DGB), coordinar las unidades del Sistema mediante la emisión de políticas y recomendaciones, así como la generación de productos y servicios de información, entre otras funciones.

Desde 1986 la DGB edita Biblioteca Universitaria, publicación técnico profesional especializada en bibliotecología y ciencia de la información, que publica artículos principalmente sobre las actividades del Sistema Bibliotecario de la UNAM (SBU), pero que está abierta también a contribuciones de otras instituciones nacionales y extranjeras.

\section{Primera época}

Biblioteca Universitaria se inicia como un boletín informativo de la DGB. Aunque el primer fascículo no consigna la fecha de edición, se ha establecido como fecha de inicio el año 1986. En la Presentación de su primer número se lee el propósito de que sirva para fortalecer la comunicación entre el sistema bibliotecario de la universidad.

La publicación nace en modestas condiciones tanto de diseño y presentación gráfica, como de trabajo editorial, con una periodicidad trimestral. Se elaboraba localmente, en formato horizontal y unida mediante grapas. La extensión de cada número era muy variable, entre las 20 y las 100 páginas. No se hace mención del tiraje ni de los medios de distribución y destino, pero si atendemos a lo que se establece en la Presentación del primer número, es de suponerse que su distribución era gratuita entre el personal de la DGB y el Sistema Bibliotecario de la Universidad. El contenido típico incluía Editorial, artículos y noticias sobre temas bibliotecarios, experiencias de trabajo, documentos y avisos.

Estas condiciones se mantienen sin cambio a lo largo de tres volúmenes (19861988), hasta que en 1989 se establece una nueva presentación en formato vertical, en mejor papel, encuadernada y que ostenta ya código ISSN. La extensión sigue siendo variable entre las 20 y 50 páginas. La naturaleza del contenido se mantiene y se aprecia la gradual introducción de algunos criterios de normalización editorial tales como: ficha catalógráfica, descripción de la publicación, directorio de funcionarios de la UNAM y la DGB, y colofón. A partir del volumen VIII (1993), se percibe otra mejora en la calidad del papel y por primera vez la publicación presenta una portada a color. Estas características persisten hasta el final del volumen IX (1994) en que concluye la primera época de la publicación. 


\section{La nueva época}

Después de una suspensión de tres años, en 1998 y a petición de académicos de la DGB, se reinicia la publicación como Nueva Época del boletín Biblioteca Universitaria, ahora con una periodicidad semestral. Se mantiene la estructura y naturaleza del contenido, pero se percibe la integración de otros elementos editoriales como mención del órgano editor, código de barras, resumen en algunos artículos, afiliación de los autores, plecas de título de la contribución y nombre de la publicación, así como fotografías, ilustraciones, gráficas y cuadros con mayor calidad y nitidez, aunque estas características eran variables en cada número.

En septiembre de 2000 se da un hecho importante con la creación del Consejo Editorial Interno, mismo que en 2006 cambia su nombre a Comité Editorial de la DGB, de acuerdo a disposiciones emitidas en ese sentido por la UNAM. Con la creación del consejo editorial se inicia una revisión profunda del estado en que se encontraba la publicación. Se decide cambiar su denominación de boletín a revista, para reflejar mejor tanto la nueva política editorial, como el rumbo hacia donde se pretendía dirigir a Biblioteca Universitaria. De aquellas reuniones, se hizo aún más evidente la necesidad de incrementar el trabajo editorial para elevar su calidad.

A partir del volumen 3, número 2, correspondiente a julio-diciembre de 2000, comienza una integración paulatina de criterios de calidad y presentación editorial a la publicación, mismos que subsisten hasta el volumen 10 correspondiente a 2007.

El contenido de un fascículo actualmente está compuesto por Presentación, artículos originales (entre cuatro y cinco), y tres secciones fijas a manera de contribuciones solicitadas a los autores: 1) Nuestros Bibliotecarios; 2) Nuestras Bibliotecas, y 3) El Sistema Bibliotecario de la UNAM en Cifras. Adicionalmente, forman parte de cada número: Reseñas de eventos, Reseñas de libros, Noticias y el anuncio del Programa de Educación Continua de la DGB.

Una de las primeras acciones del consejo editorial fue realizar una labor de normalización de la publicación. La normalización es parte medular en el campo de la información y su transferencia juega un papel estratégico que incide en cada uno de los procesos del ciclo de la información, desde su generación hasta su uso, para generar nuevos conocimientos registrados que se transformarán en documentos cognitivos (Rovalo, 2004).

\section{Diseño gráfico}

Como parte de su modernización, se consideró indispensable dotarla de una nueva imagen. Se utilizaron los servicios de una empresa de diseño que generó una nueva presentación haciendo uso de tipografía, imágenes, papel, acabados y color en las cubiertas. Este cambio surtió efecto a partir del volumen 4, número 1, enero-junio de 2001. La nueva imagen logró una presentación más clara y 
amena de los contenidos, utilizando mayor número de ilustraciones, así como papel e impresión de mejor calidad. Esta imagen ha sido bien recibida por los lectores y ha dado consistencia e identidad gráfica a la publicación. En términos económicos, se buscó que el nuevo diseño no impactara los costos, por lo que tampoco ha afectado los costos de producción.

\section{Criterios de calidad editorial}

La importancia de que las publicaciones incorporen criterios de calidad radica en que «... los editores de las revistas van tomando conciencia de que deben cumplir una serie de requisitos mínimos de calidad que inciden de forma directa en la difusión de las revistas y en su consolidación como editores de prestigio, lo cual les pone en situación favorable frente a la comunidad científica. También los bibliotecarios muestran su interés por la evaluación pues ésta se constituye en una herramienta de ayuda para la toma de decisiones en materia de suscripciones a revistas científicas" (Román, 2001).

Dada la relevancia que las revistas tienen para la comunicación científica y tomando en cuenta la ya extendida universalidad de los criterios de calidad editorial y su peso en la producción, percepción, visibilidad y evaluación de las revistas académicas, el consejo editorial consideró indispensable que Biblioteca Universitaria incluyera criterios para mejorar tanto su calidad como su estandarización. Para ello se tomó como base los criterios establecidos por el Sistema Latindex (http://www.latindex.org), no sólo por considerarlos muy completos sino porque dicho Sistema se produce en la propia DGB en colaboración con otras instituciones especializadas en información de los países ibero latinoamericanos que son miembros.

Como resultado de ese trabajo, para 2008 la revista cuenta ya con 31 de los 33 criterios considerados por el catálogo Latindex, lo que representa un 94\% de cumplimiento. En comparación, en su primera época la publicación solamente cumplía con 13 de esos criterios, es decir, un 40\% del total de criterios Latindex. Los dos criterios que no se cumplen hasta ahora son los relacionados con publicar las fechas de recepción y aceptación de originales y reforzar la apertura editorial, en el sentido de que al menos dos terceras partes del Comité Editorial sean académicos ajenos a la DGB. Además se agregaron en la cubierta, que funge como portada, todos los elementos necesarios. En la cuarta de forros se integró una tabla de contenido estructurada por artículos, secciones fijas, notas, reseñas, etc., así como el código de barras. Se mejoró y actualizó la información sobre la revista, el organismo editor, el comité editorial y las instrucciones a los autores.

\section{Publicación electrónica}

En 1998 se creó la versión electrónica de la revista, la cual incluía 20 fascículos que van de 1989 a 1994. No fueron incluidos los primeros 12 fascículos correspondientes a los tres volúmenes iniciales. En general eran documentos en HTML 
del contenido de la publicación, únicamente con texto, cuadros, sin imágenes y sin ninguna otra de las capacidades que la publicación electrónica ofrece.

Con el objetivo de presentar a los lectores una versión más apegada al formato impreso, a partir del volumen III, número 2 de la Nueva Época (2000), se inició la puesta en línea de los documentos de la revista en formato PDF. Posteriormente, iniciando con el volumen 7, número 2, de 2004 se crea una nueva versión en línea con más componentes, como una evolución del PDF que aprovecha mucho de lo ya avanzado. Esta nueva presentación retoma la entrega de artículos en HTML, (parcelados en varios tramos), además del PDF del documento completo. En HTML se proporciona el resumen, abstract y la bibliografía en ventanas separadas, y como novedades se agregan las funcionalidades sobre cómo citar cada artículo en su versión electrónica, y recomendar el artículo, ésta última a través de envío del enlace al documento por correo electrónico.

La nueva presentación resultó mucho más intensiva en tiempo de producción y en términos de horas-hombre necesarias para su producción, puesta en línea y comprobación de todos los enlaces que genera, por lo que a pesar de mejorar la presentación, no logró mantener un óptimo ritmo de actualización en el sitio web. Actualmente se está haciendo un esfuerzo por poner nuevamente la revista en línea al día, ya que no debe sacrificarse actualidad por presentación. Esta versión puede consultarse en http://dgb.unam.mx/revista/Revista\%20online/revista.html.

Una acción pendiente de implementar en el futuro sería la adopción de un sistema de administración de contenidos o CMS por sus siglas en inglés (Content Management System), que sirva para tener más control del flujo de la información en lo concerniente a la comunicación con autores y dictaminadores, el seguimiento de las contribuciones y la eventual publicación de cada fascículo. Actualmente esta acción se encuentra en evaluación.

\section{Motor de búsqueda}

Siguiendo las recomendaciones de Jakob Nielsen, se inició el estudio sobre cuál sería la mejor opción para integrar la función de búsqueda a la revista. Nielsen, basa sus consideraciones en estudios de uso y funcionalidad de sitios web, a partir de la experiencia de usuarios, concluyendo que los visitantes a un sitio web de cierta extensión y complejidad prefieren hacer uso de la función de búsqueda automatizada, generalmente proporcionada por motores de búsqueda (Nielsen, 2001).

Después de varias valoraciones se definió como mejor opción el motor de búsqueda Atomz (http://atomz.com) que constituye un motor de búsqueda externo, con capacidad de búsqueda en la sintaxis de varios idiomas, incluido el español. Es así que en 2003, ante el continuo aumento de la colección y de la complejidad que representaba tener que "hojear" cada uno de los fascículos de la revista en línea se agregó la capacidad de búsqueda automatizada, con lo cual 
se incrementó de manera sustancial la utilidad de la versión electrónica de la revista y se proporcionó, no sólo un punto de acceso más al contenido, sino uno más rápido y con mayor exactitud.

Durante las pruebas posteriores a su instalación, se encontró un alto y satisfactorio nivel de precisión y rapidez en la entrega de resultados. Según el informe correspondiente a enero de 2008 , en ese mes se tuvieron poco más de 2,000 búsquedas.

\section{Gestión editorial}

La gestión editorial de la revista es coordinada por un editor responsable, apoyado por un equipo de trabajo, todos laborando para la revista a tiempo parcial. Se realiza un trabajo muy importante de revisión y corrección con el objetivo de elevar el nivel de las contribuciones y reducir el porcentaje de rechazo. Así, se pretende estimular a la comunidad a compartir sus investigaciones y experiencias con los lectores de la revista. Como servicios adicionales, en cada artículo se realiza la normalización de la bibliografía, notas al pie y obras consultadas, así como la elaboración de resúmenes y abstracts, y asignación de palabras clave y keywords a los documentos que los requieran. Todo esto con el objetivo de cumplir con los criterios de calidad aceptados y fomentar la difusión y la inclusión de la revista en servicios especializados de información y documentación.

A partir del volumen 5 de 2002, se consiguió y se mantiene publicidad especializada de manera estable, la cual contribuye al pago de uno de los dos fascículos de cada volumen.

En cuanto a la revisión de las contribuciones, el Comité Editorial Interno formado por un grupo de académicos de la propia Dirección General de Bibliotecas, realiza una primera revisión general y apoya al editor en la selección de los revisores. Estos, son elegidos de una lista de académicos especializados en los diversos temas que abarca la revista, con adscripción institucional diversa. Cada artículo es sometido a dos revisores, siguiendo el método de doble ciego. Cuando se reciben dictámenes que no coinciden, un tercer revisor entra al desempate. Los artículos publicados están basados en su originalidad, calidad, interés y utilidad para la comunidad de lectores.

Un aspecto a remarcar es que dado el objetivo que persigue la revista y el público al que va dirigida, las contribuciones publicadas abordan principalmente experiencias de trabajo concretas, que no necesariamente son resultado de un trabajo de investigación.

\section{Análisis de las contribuciones}

Del volumen 1 de 1998 al volumen 10, número 2 de 2007, se han publicado 107 artículos, 38 reseñas de libros, 29 reseñas de eventos, 2 entrevistas, una revisión bibliográfica, dos traducciones de artículos, tres índices acumulativos, y 18 
secciones de noticias. Las contribuciones en las secciones fijas son como sigue: Nuestras Bibliotecas, 13 artículos reseñan la historia y la trascendencia de igual número de bibliotecas de la UNAM; en El Sistema Bibliotecario de la UNAM en Cifras, 13 documentos consignan cifras oficiales sobre la actividad bibliotecaria de la Universidad, y en Nuestros Bibliotecarios, se publicaron las semblanzas sobre la vida y obra de 35 profesionales de la bibliotecología de México y América Latina.

En cuanto a la procedencia de los 262 documentos publicados durante este periodo, éstos provienen de seis países: 255 de México, tres de Estados Unidos y uno de Alemania, Argentina, Chile y Francia. De los documentos mexicanos, 229 (89\%) son de la UNAM -126 de ellos (55\%) de la Dirección General de Bibliotecas- y 26 (11\%) de otras instituciones de enseñanza superior e investigación del país. Por lo que se refiere a los 285 autores de los documentos, 271 son mexicanos, seis de Argentina, cuatro de Estados Unidos y los cuatro restantes provienen de Alemania, Chile, España y Francia.

Dado que la revista ha publicado índices acumulativos, éstos fueron utilizados para el análisis de contenidos de los documentos publicados. Los temas corresponden a la clasificación de disciplinas de la base de datos CLASE (http://dgb. unam.mx/clase.html), la cual subdivide a la Bibliotecología y Ciencia de la Información en siete apartados. A esta clasificación se agregaron dos temas: Usuarios de la Información y Otros, para incluir documentos que no pudieron ser asignados en los temas originales (véase la Tabla I). En el análisis se incluyeron los artículos, secciones fijas, entrevistas, reseñas de libros, reseñas de eventos, revisión bibliográfica, índices acumulativos y traducciones. Cabe aclarar que una contribución puede ser clasificada en más de un tema.

\section{TABLA I}

\section{Clasificación temática de los documentos}

\begin{tabular}{l|c|c}
\hline \multicolumn{1}{c|}{ Tema } & Número & \% \\
\hline Análisis y sistematización de la información & 21 & 6 \\
\hline Bibliotecología y ciencia de la información & 117 & 34 \\
\hline Fuentes documentales & 40 & 12 \\
\hline Información y sociedad & 19 & 5 \\
\hline Servicios bibliotecarios y de información & 85 & 3 \\
\hline Sistemas de información & 12 & 11 \\
\hline Tecnología de la información & 38 & 4 \\
\hline Usuarios de la información & 13 & 1 \\
\hline Otros & 4 & $\mathbf{1 0 0}$ \\
\hline Total & $\mathbf{3 4 9}$ & \multicolumn{2}{|c}{} \\
\hline
\end{tabular}


De los resultados se desprende que las temáticas de interés mantienen su porcentaje de participación durante todos los años prácticamente en la misma proporción. Los artículos bajo el epígrafe "Bibliotecología y ciencia de la información" corresponden más bien a temas generales, en su mayoría reseñas biográficas de bibliotecarios distinguidos, así como artículos sobre planes de estudio de la licenciatura en bibliotecología en el país y reseñas de eventos relevantes a la profesión bibliotecaria.

Otro porcentaje importante corresponde a temas sobre servicios bibliotecarios y de información, advirtiéndose que en los últimos años aumenta el interés por abordar temas relacionados con los servicios electrónicos de información, el desarrollo de colecciones digitales en bibliotecas universitarias, las tendencias en la construcción de bibliotecas digitales, así como la cooperación bibliotecaria en el ámbito digital. La aplicación de las tecnologías de la información en las bibliotecas universitarias resultó estar en cuarto lugar en cuanto a los temas de interés publicados precedida por el estudio de fuentes documentales, que hacen referencia a archivos y colecciones especiales de fondos antiguos.

\section{Distribución y difusión}

La distribución de la revista sigue siendo mayoritariamente gratuita para la UNAM. Se tienen suscripciones, pero éstas no han sido el punto fuerte de la distribución. A partir del año 2001, se instituyeron acciones de canje y donación con otras instituciones de educación superior y bibliotecas nacionales, universitarias y públicas de México. El canje se realiza con cinco países y la donación se hace a 16 países de América y Europa.

Para aumentar la difusión de la revista, que hasta 2001 sólo era indizada en la base de datos CLASE, se realizaron acciones para que fuese incluida también en INFOBILA base de datos especializada en información y bibliotecología latinoamericana (http://cuib.unam.mx/index.htm) y COMPLUDOC, producida en la Universidad Complutense de Madrid (http://europa.sim.ucm.es/compludoc/). Asimismo, se registró en Latindex (en sus tres bases de datos: Directorio, Catálogo y Enlaces) $y$ en Ulrich's. Puesto que cumple con los criterios del Catálogo Latindex fue posible su integración a la hemeroteca virtual Redalyc a textos completos (http://redalyc.uaemex.mx).

Siendo una revista cuyo alcance es más bien nacional y latinoamericano, no es de extrañar que no aparezcan citas a ella en bases de datos como el Web of Knowledge o Scopus. En cambio, al consultar Google Scholar (http://scholar. google.com.mx/) se identificó que 16 artículos publicados en Biblioteca Universitaria han tenido 36 citas, siendo el artículo citado más antiguo uno publicado en 1994. Otro dato de interés es que en Redalyc, entre las revistas clasificadas bajo el campo temático "Ciencia de la información", Biblioteca Universitaria resulta ser la segunda con más consultas y descargas de artículos $(39,757)$ solamente superada por Anales de Documentación de la Universidad de Murcia. 


\section{Conclusiones y recomendaciones}

Es claro que las revistas de carácter técnico-profesional como Biblioteca Universitaria, tienen como objetivo difundir experiencias concretas del trabajo bibliotecario, aportando soluciones a casos prácticos, relatando experiencias de trabajo, así como aportando documentos de investigación aplicada. La orientación de la revista está claramente definida en las instrucciones a los autores, lo cual ha ayudado a definir su perfil como vehículo de divulgación de este tipo de conocimiento.

Las revistas son también un reflejo de su comunidad de lectores y autores, y Biblioteca Universitaria no es la excepción, ya que a través de sus páginas está muy bien documentado el trabajo que se realiza en el Sistema Bibliotecario de la UNAM. Es necesario subrayar aquí el importante apoyo que las bibliotecas brindan a la realización de las funciones sustantivas de la UNAM: investigación, docencia y extensión de la cultura. Por la magnitud y diversidad de este sistema bibliotecario, la información y experiencias publicadas en la revista resultan valiosas y útiles para la resolución de problemas y retos similares que enfrentan las bibliotecas del sistema universitario e incluso, otras bibliotecas del país.

En el caso de Biblioteca Universitaria, se ha hecho patente el valor de contar con un comité editorial especializado, a efecto de elevar su calidad académica. Las acciones emprendidas han permitido aumentar sustancialmente la visibilidad y uso de la publicación, así como la justificación del presupuesto que le da vida, el cual proviene principalmente de fondos públicos. Asimismo se privilegian, como sucede con muchas otras revistas latinoamericanas, las acciones de canje y donación, dado que las suscripciones y ventas son todavía marginales, aunque es deseable y factible revertir esta tendencia.

Del análisis de la procedencia de los artículos, se desprende que la revista aún sigue cumpliendo con su objetivo inicialmente declarado de servir como vehículo de comunicación para la comunidad bibliotecaria de la UNAM. No obstante, la revista debe aspirar a publicar, cada vez más, experiencias de otras instituciones.

Además de incorporar los criterios de calidad Latindex que aún no se cumplen, la revista debe integrar un comité editorial con representación interinstitucional e internacional, así como apuntalar su inclusión en más servicios referenciales, de resúmenes y textos completos.

También sería recomendable adoptar para la gestión de la revista un sistema de administración de contenidos, que permita mejorar el trabajo editorial. Se han estudiado los que hay disponibles actualmente y el personal involucrado ha tomado algunos cursos de capacitación en esta área, a efecto de realizar una implantación orientada y exitosa.

En suma, con el trabajo editorial realizado hasta ahora, Biblioteca Universitaria enfrentará su segunda década de existencia fortalecida y con una mayor visibilidad y presencia en el área de las bibliotecas universitarias en México. 


\section{Bibliografía}

Alonso Gamboa, J. O. (2002): Evaluación de revistas científicas en América Latina: propuesta de un modelo para su uso en bases de datos. México: el autor, 2002, 246 p. Tesis maestría (Maestría en bibliotecología) - UNAM, Facultad de Filosofía y Letras.

Atomz (1999): Atomz site Search. Disponible en: http://atomz.com/ [consulta: 10 abril 2008].

Centro Universitario de Investigaciones Bibliotecológicas-UNAM. Infobila. Disponible en: http://cuib.unam.mx/index.htm [consulta: 3 abril 2008].

Dirección General de Bibliotecas-UNAM (1996): Base de datos CLASE. Disponible en http://dgb.unam.mx/clase.html [consulta: 3 abril 2008].

Dirección General de Bibliotecas-UNAM (1996): Latindex. Disponible en: http://www.latindex.org/ [consulta: 10 abril 2008].

Google. Google Scholar. Disponible en: http://scholar.google.com.mx [consulta 26 junio 2008].

Nielsen, J. (2001): Search: visible and simple. Alertbox. Disponible en: http://www.useit. com/alertbox/20010513.html [consulta: 10 abril 2008].

Proquest. Ulrich's Periodiclas Directory. Disponible en: http://www.ulrichsweb.com [consulta: 24 junio 2008].

Román Román, A. (Coord.) (2001): La edición de revistas científicas: guia de buenos usos. Madrid. Centro de Información y Documentación Científica. ISBN 84-00-07916-7.

Rovalo de Robles, M. L. (2004): Normalización de Revistas Científicas. Acta Universitaria, vol. $14, \mathrm{n}^{\circ} 3$, p. 5-11. Disponible en: http://redalyc.uaemex.mx/redalyc/src/inicio/ ArtPdfRed.jsp?iCve=41614301\&iCveNum=1785 [consulta: 10 abril 2008].

Silva, L. A. G., Almeida, R. L., Paranhos, B. S. y Florentino, C. A. A. (1996): Buscando soluçoes para se publicar na Internet: a experiencia do IBICT com a Ciencia de Informaçao on-line. Ciencia da Informaçao, vol. 25, n $\mathrm{n}^{\circ}$ 3, p. 454-460. Disponible en: http://revista.ibict.br/index.php/ciinf/article/view/473/432 [consulta: 10 abril 2008].

Universidad Autónoma del Estado de México. Redalyc. Disponible en: http://redalyc. unaemex.mx [consulta: 24 junio 2008].

Universidad Complutense de Madrid. Compludoc. Disponible en: http://europa.sim.ucm. es/compludoc/ [consulta: 3 abril 2008]. 